\title{
Are Surgical and Non-Operating Room Interventions Safe in the COVID-19 Pandemic?: A Retrospective Study
}

\section{Serap Aktas Yildirim ( $\nabla$ serapaktas79@yahoo.com.tr )}

Acibadem Mehmet Ali Aydinlar University School of Medicine

\section{Zeynep Sarikaya}

Acibadem Mehmet Ali Aydinlar University School of Medicine

\section{Halim Ulugol}

Acibadem Mehmet Ali Aydinlar University School of Medicine

\section{Sanem Ozata}

Acıbadem University Atakent Hospital

\section{Ugur Aksu}

Istanbul University Faculty of science

\section{Fevzi Toraman}

Acibadem Mehmet Ali Aydinlar University School of Medicine

\section{Research Article}

Keywords: COVID-19, Surgery, Nonoperating room intervention, Mortality, Intensive care requirement, COVID-19 transmission

Posted Date: December 3rd, 2020

DOI: https://doi.org/10.21203/rs.3.rs-110458/v1

License: (c) (i) This work is licensed under a Creative Commons Attribution 4.0 International License. Read Full License 


\section{Abstract}

Background It is not known whether emergency surgeries and interventions are safe during the pandemic period. We investigate the effect of surgery and intervention on COVID-19 disease progression, intensive care (ICU) need, mortality, and virus transmission to patients and healthcare workers.

Methods This retrospective study was conducted between 20 March -20 May 2020 in six hospitals in Istanbul. Patients were examined in three groups. Group I patients who underwent emergency surgery, Group II patients who underwent emergency nonoperating room intervention, Group III patients who underwent inpatient COVID-19 treatment but did not have any surgery or intervention. During this period, all healthcare workers and patients diagnosed with COVID-19 with any of the symptoms, PCR positivity, or Thorax CT findings were recorded. Patient and healthcare worker transmission, unplanned intensive care need, and death due to COVID-19 were also recorded.

Results 1,273 surgical, 476 nonoperating room intervention patients, and 1,884 COVID-19 inpatients were examined. ICU requirement of COVID-19 patients who had surgery was 20 times higher than COVID-19 inpatients and intervention patients but there is no difference in mortality. The total mortality rates were $2.3 \%$ in surgical patients, $3.3 \%$ in intervention patients diagnosed with COVID-19, and 3\% in COVID-19 inpatients. COVID-19 PCR positivity among hospital workers was \% 2.4 . Only $3.3 \%$ of the infected frontline healthcare workers were anesthesiologists. No deaths occurred among infected healthcare workers.

Conclusions Emergency surgeries and interventions during the pandemic period did not increase mortality and can be performed safely with very low transmission rates by complying with the effective use of personal protective equipment.

\section{Introduction}

Due to the high contagion pattern of this SARS-CoV-2 and the rapid increase in the number of cases, a pandemic was declared by the WHO. In most of the countries, elective cases have been cancelled, and only urgent cases have been addressed during this period. Some guidelines recommend symptom screening and nucleic acid amplification testing (PCR) before nonemergency procedures, but there is insufficient data on how to make decisions in emergency cases. ${ }^{1}$ Due to the low sensitivity of tests and high levels of asymptomatic carriers, it is difficult to detect a patient who is infected with SARS-CoV-2. ${ }^{2}$ Therefore, healthcare workers are also at risk in terms of disease transmission. Limited studies have shown that patients infected with SARS-CoV-2 have higher perioperative mortality and morbidity because of rapid ARDS progression and cardiovascular failure. ${ }^{3}$

In the absence of essential surgical treatment, patients who do not receive the necessary health care may become more vulnerable to coronavirus infection. Cancellation or postponement of most cases during the pandemic period may cause more harm to patients than the damage caused by the SARS-CoV-2 in the long term. ${ }^{4-6}$ Precisely for these reasons, the necessity emerges of understanding the safety of emergency surgery and intervention applications for both healthcare professionals and patients in pandemic hospitals. As a health group that follows and treats patients diagnosed with COVID-19 as well as performing emergency surgery and interventions in our own hospitals, we tried to determine whether this path we follow will be safe in the later peaks of the pandemic.

\section{Method}

\section{Overview}

This retrospective study was conducted between 20 March and 20 May 2020 in six hospitals in Istanbul that belong to the Acibadem Health Group and Acibadem MAA University. Ethical approval was obtained from the Regional Ethical Committee of Acibadem MAA University (protocol no:ATADEK-2020/08). Informed consent was obtained from all patients. This study assesses the effects of emergency surgery and interventions performed during the pandemic period on COVID-19 disease 
progression and the risk of transmission to healthcare workers and patients. The authors are responsible for the accuracy and completeness of the data.

\section{Patients}

Patients who underwent emergency surgery and non-operating room interventions were admitted to the study. All hospital workers and all patients who underwent COVID-19 treatment but did not have any surgery or intervention in the same period were included. Those under 18 years of age were excluded.

\section{Trial procedures}

Patients were examined in three groups. Group I patients underwent emergency surgery, Group II patients underwent emergency nonoperating room intervention and Group III patients underwent inpatient COVID-19 treatment but did not have any surgery or intervention. Accompanying comorbidities, epidemiological, clinical, laboratory, and radiological data, COVID-19 suggestive symptoms, anaesthesia management, surgical type, intensive care and mechanical ventilation needs and outcome data were recorded. PCR was studied from symptomatic patients and healthcare workers. Diagnosis of COVID-19 was made based on PCR positivity, chest computerized tomography (CT) or the presence of clinical symptoms. In all patients diagnosed with COVID-19, supportive treatment and antiviral therapy were initiated in the preoperative period. During this period, all healthcare workers diagnosed with COVID-19 were recorded. Patient and healthcare worker transmission, unplanned intensive care need and death due to COVID-19 were recorded. Unplanned intensive care unite (ICU) need is defined as admission to intensive care (not related to surgical intervention) within the first 10 days after surgery or intervention in patients who do not come from the ICU. All patients who were diagnosed with COVID-19 and started treatment in the postoperative period were accepted as in-hospital transmission, since it is not known whether the patients were admitted to the procedures in the asymptomatic or presymptomatic period.

\section{Objectives}

The primary objective is to evaluate the risk of virus transmission to patients and healthcare workers in emergency surgery and intervention at pandemic hospitals. The secondary objective is to examine the effect of surgery and intervention on COVID-19 disease progression, ICU need and mortality. The third objective is to demonstrate the safety of performing emergency surgeries and interventions during this pandemic period.

\section{Statistical Analysis}

All data were analysed using RStudio Cloud 3.5.2 Eggshell Igloo. The Shapiro Wilk Normality Test was used to detect normal distributions. The data were presented as percentages and medians (minimum-maximum). A chi-square test was used to compare categorical variables whereas a Mann-Whitney $U$ test was used to compare numerical variables. A Wilcoxon signedrank paired test was used to compare preoperative and postoperative laboratories in COVID-19 patient subgroups. A p-value $<0.05$ was considered statistically significant.

\section{Results}

\section{Clinical characteristics of the patients}

1,273 surgical, 476 intervention patients and 1,884 inpatients with a diagnosis of COVID-19 were examined. When we compared the COVID-19 inpatients and the patients who had surgery and intervention, ASA scores were significantly higher in surgery and intervention patients. Patient characteristics and demographic data are shown in Table 1.

It was determined that the most common emergency surgery was cesarean section (33.7\%), and the most common intervention was gastrointestinal endoscopy (49.8\%). General anaesthesia was applied to $64.3 \%$ of surgical patients, while all interventions were applied with sedoanalgesia. 


\section{Disease prevalence and PCR test results}

In the pre-operative period, $232(18.1 \%)$ of 1,273 patients who underwent surgery were examined with PCR, 15 (6.5\%) of these patients had positive results. $110(23.1 \%)$ out of 476 patients who underwent interventions were examined with PCR, while only $2(18 \%)$ of these patients had positive results. The prevalence of COVID-19 during the preoperative period was $3.1 \%$ in surgical patients and $5.9 \%$ in intervention patients. In the postoperative period, it was $0.3 \%$ in surgical patients and $0.4 \%$ in intervention patients.

92\% of COVID-19 inpatients were screened by PCR test, and 12.8\% of them were PCR positive. PCR data and symptoms of all patients in the perioperative period are shown in Table 2, and the perioperative laboratory data are shown in Table 3.

\section{ICU need and mortality}

The ICU needs of surgical patients diagnosed with COVID-19, intervention patients diagnosed with COVID-19 and COVID-19 inpatient were determined to be $44.2 \%, 20 \%$ and $24.4 \%$, respectively. The total mortality rate was $2.3 \%$ in surgical patients, $3.3 \%$ in intervention patients diagnosed with COVID-19 and 3\% in COVID-19 inpatients. ICU need and mortality data are shown in Table 4.

\section{Infected patients and healthcare workers}

In our 968-bed healthcare group, a total of 5,874 healthcare workers, $20.3 \%$ of whom were doctors and $39 \%$ of whom were auxiliary staff, worked during the pandemic period.

Total of 9,889 PCR tests were performed in hospitals, and the positivity rate was $12.8 \%$. Hospital staff received a total of 1,824 tests, and PCR positivity was detected as $7.8 \%$. No deaths occurred among infected healthcare workers. In this period, the prevalence of COVID-19 PCR positivity among hospital workers was $2.4 \%$.

The number of contaminants and disease prevalence among the healthcare workers and infected patients is shown in Table 5.

\section{Discussion}

SARS-CoV-2 is highly contagious and can be transmitted in the form of aerosol. Both healthcare professionals and patients are at risk during surgery and interventions. This has become an important problem, especially for healthcare institutions that treat patients diagnosed with COVID-19 and follow up on other patients at the same time.

In light of the information in the literature, we made the following preparations to become a pandemic hospital in our health group. COVID-19 operating rooms have been allocated for COVID-19 cases with at least one negative-pressure room for case inputs and outputs. In all confirmed or suspected cases, personal protective equipment( PPE) and special intubation boxes were used for aerosol-generating procedures.

All healthcare workers with symptoms of COVID-19, regardless of whether a PCR test or CT was performed, were taken into isolation for 14 days to limit the exposure of healthy individuals to Coronavirus.

As a healthcare group, we have tried to determine whether this path we follow will be safe in the later peaks of the pandemic.

There was a significant difference between the preoperative COVID-19 prevalence of surgery and intervention patients, and we attributed this to the presence of young cesarean patients without chronic disease who did not need to stay in hospital for a long time. Therefore, we found that the average age of surgical patients was quite low compared to patients undergoing interventions and COVID-19 inpatients ( $p<0.001)$. However, the high COVID-19 ratio in the interventional patients was associated with the presence of fragile, hospitalized patients with more risk factors. For this reason, during the preoperative period, the intervention patients were given more PCR tests and thorax CT was more frequently taken. 
Patients with COVID-19 who underwent surgery were found to be significantly different in their advanced age, male bodies, HT and use of immunosuppressive drugs when compared with those not diagnosed with COVID-19. The need of these patients for postoperative intensive care was 20 times higher than for those who were negative, but there was no significant difference in mortality $(p>0.05)$. We think that the secondary impact created by surgery increases the need for intensive care, but that antiviral and supportive treatment initiated before surgery has a positive effect on mortality.

We found a similar relationship in mortality for COVID-19-diagnosed intervention patients pre-operatively, but half of the surgery group needed the ICU. Intervention patients did not experience any secondary trauma caused by surgery like surgical patients did, and all patients underwent sedoanalgesia throughout the procedure. However, while thorax CT, which was taken in the pre-operative period, showed more pneumonia in the intervention patients, we found that these patients used more immunosuppressive drugs compared to the surgical patients, despite the lesser ICU need. We have seen that interventions performed with sedoanalgesia that do not require endotracheal intubation in COVID-19 patients do not increase the need for intensive care and mortality. Therefore, we think that interventions requiring sedoanalgesia can be performed more safely in patients with COVID-19 compared to surgery. The guideline published by the American College of Surgeons in March, when the pandemic was at its peak, recommended the implementation of non-surgical treatments to delay or prevent the need for surgery and postpone non-critical procedures. If intervention is safer than surgery for COVID-19 patients, it can be applied more safely as an alternative method, especially in patients at risk of having surgery in other peaks of the pandemic. ${ }^{7}$

To understand how important the secondary impact of surgery is, we compared COVID-19 patients who had undergone surgery or interventions with those hospitalized for COVID-19 without any surgical procedure or intervention. Although the ages are similar, we found that inpatients had fewer risk, that there was no significant difference in mortality, and that the need for intensive care was found to be higher in patients who underwent surgery compared to other patients. All these results show that secondary trauma such as surgery will negatively affect the clinical course of COVID-19 patients. The study, which analyzed 1,128 surgical patients infected with SARS-CoV-2, showed this secondary impact caused by surgery. Mortality was $16.3 \%$ in those who had minor surgery, while the rate was $26.9 \%$ in those who underwent major surgery. ${ }^{8}$

When we look at the patients diagnosed with COVID-19 who underwent intervention and surgery, we see that the incidence of cancer is higher than for those COVID-19 inpatients. Therefore, we found that the use of immunosuppressive drugs in these patients was significantly higher. Cancer and immunosuppressive drug use may be risk factors when compounded with surgery and COVID-19. Cancer patients are considered more susceptible to infections due to both the malignancy and the immunosuppressive state that results from anticancer treatments. ${ }^{9}$ If there is time, screening cancer patients that require emergency surgery and intervention for COVID-19 with diagnostic methods such as symptoms, PCR and thorax CT may direct the treatment, especially during the pandemic period. A study from China showed that patients with cancer had a five-fold higher risk of COVID-19 infection, a higher risk of serious events, and a greater need for intensive care help than cancer-free patients. ${ }^{10}$ In Italy, only $0.71 \%$ of 1,257 cancer patients who did not undergo surgery during the peak of the pandemic but received active anticancer treatment in the hospital were diagnosed with COVID-19; the mortality rate was $22 \%{ }^{11}$ The mortality of our patients is seen to be quite low compared to this study, but it should not be forgotten that the prevalence of COVID-19 in the patients who underwent intervention, a significant portion of whom were immunosuppressive patients, was approximately 2 times higher than in those who had surgery. However, it should be understood that they are a patient group that needs to be more careful in terms of transmission due to their fragility, concomitant comorbidities and long-term inpatient treatment. ${ }^{12}$

In the first stage of the pandemic, our pre-operative PCR test rate was very low, access to the test kit was limited and the result time was long. Therefore, only clinically suspicious cases could be tested in an emergency setting. Although the rate of preoperative positive results was low, diagnosis of thorax BT and clinical symptoms became more prominent and treatment was required in more patients. We found that the pre-operative PCR test alone was not a guide in our patients. Because, while $6.5 \%$ of the tests performed were positive, $22.5 \%$ of thorax CTs had infiltration, even in intervention patients this rate was $30.8 \%$. Six publications in the literature on the use of chest CT in pre-operative elective and emergency situations for COVID-19 screening were reviewed by Agrawal et al. CT positivity was 10-80\% (range 4.81-16.8), while PCR positivity was 1-88\% (range 0-8.81). found. As a result, they showed that although it is not accepted as a gold standard, the positive predictive value of CT 
increased in regions with high prevalence. ${ }^{13}$ In our cases, the rate of patients diagnosed with Chest CT was higher. We think that the use of CT is an important screening test in high risk patients who will undergo emergency surgery and intervention.

Liu et al. have shown that NLR is an early-stage predictive factor for detecting COVID-19 infected patients. ${ }^{14}$ When the neutrophil/lymphocyte ratios and lymphocyte values are compared in the surgical and intervention patients diagnosed with pre-operative COVID-19, we see that they are significant. Thus, they can be used as auxiliary diagnostic tests in cases where PCR and CT are not accessible.

Our patient transmission prevalence for both groups is lower than $2 \%$, which is the low prevalence value determined by the $\mathrm{WHO}$, and the interventions and surgeries were performed in our hospital with a low risk of contamination. ${ }^{15}$

According to the Chinese study, $3.8 \%$ of confirmed cases were healthcare workers, while only five resulted in death. ${ }^{16} 4.3 \%$ of confirmed cases in our hospitals were frontline healthcare workers. These numbers seem quite high, but at the beginning of the pandemic, the shortage and improper use of personal protective equipment was a problem. COVID-19 transmission occurred in $142(2.4 \%)$ of 5,874 healthcare workers, and there was no mortality. Only $3.3 \%$ of the infected frontline healthcare workers were anesthesiologists. We see that the infection and contamination rates of the doctors and nurses working in the ward are three times higher, which shows that the healthcare professionals working in the operating room and the intervention areas use personal protective equipment more frequently and correctly.

Our study has some limitations. The SARS-CoV-2 PCR test could not be performed on most of the patients due to the insufficiency of the test kit, and only symptom screening was performed, so we may not have identified asymptomatic infected patients. Some of our patients were young without comorbidity; even if they were infected, they may have had an asymptomatic pneumonia progression. The low number of surgical and intervention patients infected with SARS-CoV-2 decreases the statistical power, and this is an important limiting factor of our study. Therefore, multicentric studies with a much higher number of patients are needed.

\section{Conclusion}

It should be known that it is very important to scan surgical and intervention patients for COVID-19 using all possible diagnostic methods in the pre-operative period, especially with immunosuppressive patients; that surgery performed on patients will double their ICU need; and that the secondary impact caused by interventions on the progression of COVID-19 infection is very low. Patients with COVID-19 who underwent surgery and intervention with antiviral and supportive treatment initiated in the preoperative period did not have significantly higher mortality than COVID-19 inpatients who did not have surgery or intervention. In the operating rooms and invasive intervention areas with a relatively low density, we can say that due to the more regular use of the necessary personal protective equipment, there are fewer contaminants, and the doctors and nurses working in the emergency rooms and wards have higher transmission rates.

If your health system has enough facility capacity and capability, you can proceed with the essential surgical procedures and interventions with area safety rules and effective use of PPE during pandemic.

\section{Abbreviations}

COVID-19: Corona Virus Disease 2019; PPE: personal protective equipment; WHO: World Health Organization; ICU: Intensive care unit; PCR: Polymerase chain reaction; CT: Computerized tomography; ARDS: Adult Respiratory Distress syndrome

\section{Declarations}

\section{Authors' contributions}


SAY, FT, HU and ZTS designed the study. SAY drafted the manuscript. ZTS modified the manuscript. SAY, ZTS, HU and COVID19 study group performed the study and collected data. BG performed the statistical analysis. All authors read and approved the final manuscript.

\section{Funding}

There was no funding and financial support for this study.

\section{Availability of data and materials}

The datasets during the current study available from the corresponding author on reasonable request.

\section{Ethics approval and consent to participate}

Ethical approval was obtained from the Regional Ethical Committee of Acibadem MAA University (protocol no: ATADEK2020/08) and therefore been performed in accordance with the ethical standards laid down in the 1964 Declaration of Helsinki and its later amendments. The written informed consent was obtained from all patients.

\section{Consent for publication}

Not applicable.

\section{Competing interests}

The authors declare that they have no competing interests.

\section{Acknowledgements}

Not applicable

\section{References}

1. The ASA and APSF Joint Statement on Perioperative Testing for the COVID-19 Virus. Available at:The ASA and APSF Joint Statement on Perioperative Testing for the COVID-19 Virus Accessed September 1, 2020.

2. Wang W, Xu Y, Gao R, Lu R, Han K, Wu G, Tan W. Detection of SARS-CoV-2 in Different Types of Clinical Specimens. JAMA 2020.

3. Lei S, Jiang F, Su W, Chen C, Chen J, Mei W, Zhan L-Y, Jia Y, Zhang L, Liu D, Xia Z-Y, Xia Z. Clinical characteristics and outcomes of patients undergoing surgeries during the incubation period of COVID-19 infection. EClinicalMedicine 2020;21:100331.

4. Stahel PF. How to risk-stratify elective surgery during the COVID-19 pandemic? Patient Saf Surg 2020;14:8.

5. Grass F, Behm KT, Duchalais E, Crippa J, Spears GM, Harmsen WS, Hübner M, Mathis KL, Kelley SR, Pemberton JH, Dozois EJ, Larson DW. Impact of delay to surgery on survival in stage I-III colon cancer. Eur J Surg Oncol 2020;46:455-61.

6. Shin DW, Cho J, Kim SY, Guallar E, Hwang SS, Cho B, Oh JH, Jung KW, Seo HG, Park JH. Delay to curative surgery greater than 12 weeks is associated with increased mortality in patients with colorectal and breast cancer but not lung or thyroid cancer. Ann Surg Oncol 2013;20:2468-76.

7. Surgeons AC of. COVID-19: elective case triage guidelines for surgical care. 2020.

8. COVIDSurg Collaborative. Mortality and pulmonary complications in patients undergoing surgery with perioperative SARSCoV-2 infection: an international cohort study. Lancet 2020;396:27-38.

9. Kamboj M, Sepkowitz KA. Nosocomial infections in patients with cancer. Lancet Oncol 2009;10:589-97.

10. Liang W, Guan W, Chen R, Wang W, Li J, Xu K, Li C, Ai Q, Lu W, Liang H, Li S, He J. Cancer patients in SARS-CoV-2 infection: a nationwide analysis in China. Lancet Oncol 2020;21:335-7. 
11. Omarini C, Maur M, Luppi G, Narni F, Luppi M, Dominici M, Longo G, Piacentini F. Cancer treatment during the coronavirus disease 2019 pandemic: Do not postpone, do it! Eur J Cancer 2020;133:29-32.

12. Zhang L, Zhu F, Xie L, Wang C, Wang J, Chen R, Jia P, Guan HQ, Peng L, Chen Y, Peng P, Zhang P, Chu Q, Shen Q, Wang Y, Xu SY, Zhao JP, Zhou M. Clinical characteristics of COVID-19-infected cancer patients: a retrospective case study in three hospitals within Wuhan, China. Ann Oncol 2020;31:894-901.

13. Agrawal V, Yadav SK, Sharma D. Pre-operative CT Chest as a screening tool for COVID-19: An appraisal of current evidence. BJS (British Journal of Surgery) 2020;n/a. Available at: https://doi.org/10.1002/bjs.12039.

14. Liu J, Liu Y, Xiang P, Pu L, Xiong H, Li C, Zhang M, Tan J, Xu Y, Song R, Song M, Wang L, Zhang W, Han B, Yang L, Wang X, Zhou G, Zhang T, Li B, Wang Y, Chen Z, Wang X. Neutrophil-to-lymphocyte ratio predicts critical illness patients with 2019 coronavirus disease in the early stage. J Transl Med 2020;18:206.

15. Hanson KE, Caliendo AM, Arias CA, Englund JA, Lee MJ, Loeb M, Patel R, El Alayli A, Kalot MA, Falck-Ytter Y, Lavergne V, Morgan RL, Murad MH, Sultan S, Bhimraj A, Mustafa RA. Infectious Diseases Society of America Guidelines on the Diagnosis of COVID-19. Clin Infect Dis 2020. Available at: http://dx.doi.org/10.1093/cid/ciaa760.

16. Wu Z, McGoogan JM. Characteristics of and important lessons from the coronavirus disease 2019 (COVID-19) outbreak in China: summary of a report of 72314 cases from the Chinese Center for Disease Control and Prevention. JAMA 2020;323:1239-42.

\section{Tables}

Table 1. Patients characteristics

\begin{tabular}{|c|c|c|c|c|c|c|c|}
\hline & $\begin{array}{l}S P \\
(n=1273)\end{array}$ & $\begin{array}{l}\text { IP } \\
(n=476)\end{array}$ & $\begin{array}{l}\text { Total }(\mathrm{SP}+ \\
\text { IP }) \\
(\mathrm{n}=1749)\end{array}$ & $\begin{array}{l}\text { HCovP } \\
(n=1884)\end{array}$ & $\mathrm{p}_{1}$ & $\mathrm{p}_{2}$ & $\mathrm{p}_{3}$ \\
\hline Age, years & $\begin{array}{l}37(32- \\
50)\end{array}$ & $\begin{array}{l}47(36- \\
60)\end{array}$ & $39(32-55)$ & $52(38-67)$ & $<0.001$ & $<0.001$ & $<0.001$ \\
\hline Male, $\mathrm{n}(\%)$ & $357(28.0)$ & $193(40.6)$ & $550(31.4)$ & $1036(54.9)$ & $<0.001$ & $<0.001$ & $<0.001$ \\
\hline $\begin{array}{l}\text { ASA Classification }>2, n \\
(\%)\end{array}$ & $115(9.0)$ & $61(12.8)$ & $176(10.1)$ & 35 (1.9) & $<0.001$ & $<0.001$ & $<0.001$ \\
\hline \multicolumn{8}{|l|}{ Comorbidities, n (\%) } \\
\hline Hypertension & $201(15.8)$ & $140(29.4)$ & $341(19.5)$ & $206(10.9)$ & $<0.001$ & $<0.001$ & $<0.001$ \\
\hline DM & $110(8.6)$ & $56(11.8)$ & $166(9.5)$ & $114(6.1)$ & 0.006 & $<0.001$ & $<0.001$ \\
\hline CRF & $26(2.0)$ & $11(2.3)$ & $37(2.1)$ & $32(1.7)$ & 0.482 & 0.372 & 0.359 \\
\hline CAD & $77(6.0)$ & $66(13.9)$ & $143(8.2)$ & $77(4.1)$ & 0.012 & $<0.001$ & $<0.001$ \\
\hline Immunosuppressive drug & $81(6.4)$ & $94(19.8)$ & $175(10.0)$ & $3(0.2)$ & $<0.001$ & $<0.001$ & $<0.001$ \\
\hline COPD & $32(2.5)$ & $42(8.8)$ & $74(4.2)$ & $37(2.0)$ & 0.302 & $<0.001$ & $<0.001$ \\
\hline Smoking & $218(17.1)$ & $92(19.3)$ & $310(17.7)$ & 318 (16.9) & 0.864 & 0.208 & 0.506 \\
\hline CVA & $18(1.4)$ & $10(2.1)$ & $28(1.6)$ & $18(1.0)$ & 0.235 & 0.039 & 0.082 \\
\hline CoVID (+) patients, n (\%) & $43(3.4)$ & $30(6.3)$ & $73(4.2)$ & $1884(100)$ & $<0.001$ & $<0.001$ & $<0.001$ \\
\hline
\end{tabular}

CAD, coronary arterial disease; COPD, chronic obstructive pulmonary disease; CRF, chronic renal failure; CVA, cerebrovascular accident; DM, diabetes mellitus; HCovP, hospitalized COVID patients; IP, intervention patients; SP, surgical patients; $\mathrm{p}_{1}$, SP\&HCovP ; $p_{2}$, IP\&HCovP ; $p_{3}$, Total\&HCovP 
Table 2.Patients PCR data, symptoms and prevalence 


\begin{tabular}{|c|c|c|}
\hline & $\begin{array}{l}S P \\
(n=1273)\end{array}$ & $\begin{array}{l}\text { IP } \\
(n=476)\end{array}$ \\
\hline \multicolumn{3}{|l|}{ Preoperative Period } \\
\hline \multicolumn{3}{|l|}{ The number of PCR test, $\mathrm{n}(\%)$} \\
\hline total & $232(18.2)$ & $110(23.1)$ \\
\hline positive & $15(6.5)$ & $2(1.8)$ \\
\hline \multicolumn{3}{|l|}{ The number of $\mathrm{CT}, \mathrm{n}(\%)$} \\
\hline total & $151(11.9)$ & $78(16.4)$ \\
\hline positive infiltration & $34(22.5)$ & $26(30.8)$ \\
\hline Patients who have only clinical symptoms, n (\%) & $4(0.3)$ & $0(0.2)$ \\
\hline Treated patients, n (\%) & $39(3.1)$ & $28(5.9)$ \\
\hline Prevalence (\%) & 3.1 & 5.9 \\
\hline \multicolumn{3}{|l|}{ Symptoms, n (\%) } \\
\hline Cough & $18(46.2)$ & $7(28.0)$ \\
\hline Fever & $14(35.9)$ & $5(20.0)$ \\
\hline Arthralgia & $8(20.5)$ & $3(12.0)$ \\
\hline Throat ache & $3(7.7)$ & $1(4.0)$ \\
\hline Neurological findings & $2(5.1)$ & $1(4.0)$ \\
\hline Diarrhea & $2(5.1)$ & $1(4.0)$ \\
\hline Anosmia & $1(2.6)$ & $0(0.0)$ \\
\hline \multicolumn{3}{|l|}{ Postoperative Period } \\
\hline & SP & $\mathrm{IP}$ \\
\hline & $(n=1273)$ & $(n=476)$ \\
\hline \multicolumn{3}{|l|}{ The number of PCR test, $n$ (\%) } \\
\hline total & $47(3.7)$ & $18(3.8)$ \\
\hline positive & $2(4.3)$ & $2(11.1)$ \\
\hline \multicolumn{3}{|l|}{ The number of CT, $\mathrm{n}(\%)$} \\
\hline total & $19(3.0)$ & $18(4.2)$ \\
\hline positive infiltration & $3(15.8)$ & $1(5.6)$ \\
\hline Patients who have only clinical symptoms, n (\%) & $1(0.1)$ & $0(0.0)$ \\
\hline Treated patients, n (\%) & $4(0.3)$ & $2(0.4)$ \\
\hline Prevalence (\%) & 0.3 & 0.4 \\
\hline \multicolumn{3}{|l|}{ Symptoms, n (\%) } \\
\hline Cough & $3(75.0)$ & $2(100.0)$ \\
\hline Fever & $2(50.0)$ & $1(50.0)$ \\
\hline Arthralgia & $1(25.0)$ & $1(50.0)$ \\
\hline
\end{tabular}

Page 10/14 


\begin{tabular}{|c|c|c|}
\hline Throat ache & $0(0.0)$ & $0(0.0)$ \\
\hline Neurological findings & $0(0.0)$ & $0(0.0)$ \\
\hline Diarrhea & $0(0.0)$ & $0(0.0)$ \\
\hline Anosmia & $0(0.0)$ & $0(0.0)$ \\
\hline
\end{tabular}

CT, computed tomography; IP, intervention patients; PCR, polymerase chain reaction; SP, surgical patients Table 3. Comparison of COVID (-) and COVID (+) Surgical and Intervention Patients 


\begin{tabular}{|c|c|c|c|c|c|c|}
\hline & $\begin{array}{l}\text { COVID (-) SP } \\
(n=1234)\end{array}$ & $\begin{array}{l}\text { Preop COVID }(+) \\
\text { SP } \\
(n=39)\end{array}$ & $\mathrm{p}_{1}$ & $\begin{array}{l}\operatorname{COVID}(-) \text { IP } \\
(n=446)\end{array}$ & $\begin{array}{l}\text { Preop COVID }(+) \\
\text { IP } \\
(n=28)\end{array}$ & $\mathrm{p}_{2}$ \\
\hline Age, years & $37(32-48)$ & $57(41-66)$ & $<0.001$ & $46(35-59)$ & $62(51-72)$ & 0.001 \\
\hline Male, n (\%) & 337 (27.3) & $21(53.9)$ & 0.002 & $178(39.9)$ & $15(53.6)$ & 0.060 \\
\hline $\mathrm{BMI}, \mathrm{kg} / \mathrm{m}^{2}$ & $25.7(21.8-29.1)$ & $26.9(22.8-30.1)$ & 0.451 & $26.2(23.1-29.0)$ & $26.7(25.0-28.7)$ & 0.599 \\
\hline $\begin{array}{l}\text { ASA } \\
\text { classification }>2, n \\
(\%)\end{array}$ & $813(65.9)$ & $30(76.9)$ & 0.120 & $323(70.7)$ & $20(80.0)$ & 0.438 \\
\hline \multicolumn{7}{|l|}{$\begin{array}{l}\text { Preoperative } \\
\text { comorbidities, n (\%) }\end{array}$} \\
\hline Hypertension & $106(8.6)$ & $5(12.8)$ & 0.302 & $51(11.4)$ & $6(21.4)$ & 0.107 \\
\hline Divi & $24(2.0)$ & $3(7.7)$ & 0.052 & $9(2.0)$ & $3(10.7)$ & 0.013 \\
\hline car & $74(6.0)$ & 4 (10.3) & 0.416 & $58(13.0)$ & $9(32.1)$ & 0.003 \\
\hline CAD & $72(5.8)$ & $9(23.1)$ & $<0.001$ & 86 (19.3) & $7(25.0)$ & 0.383 \\
\hline drugs & $24(2.0)$ & $8(20.6)$ & $<0.001$ & $35(7.8)$ & $7(25.0)$ & 0.002 \\
\hline COPD & $206(16.7)$ & $12(30.8)$ & 0.028 & $87(19.5)$ & $5(17.9)$ & 1.000 \\
\hline $\begin{array}{l}\text { Smoking } \\
\text { CVA }\end{array}$ & $15(1.2)$ & $3(7.7)$ & 0.006 & $8(1.8)$ & $2(7.1)$ & 0.157 \\
\hline $\begin{array}{l}\text { Preoperative } \\
\text { laboratories }\end{array}$ & $8.6(6.8-10.9)$ & $7.7(5.9-10.7)$ & 0.018 & $7.7(6.0-9.9)$ & $7.7(5.9-10.7)$ & 0.911 \\
\hline VVDC & $1.84(1.43-2.33)$ & $1.30(0.80-1.90)$ & $<0.001$ & $1.72(1.22-2.32)$ & $1.30(0.80-1.90)$ & 0.006 \\
\hline NLCR & $3.2(2.0-4.7)$ & $5.5(3.0-13.5)$ & $<0.001$ & $2.8(1.9-4.8)$ & $5.4(2.9-9.4)$ & 0.011 \\
\hline $\begin{array}{l}\text { Postoperative } \\
\text { laboratories }\end{array}$ & $11.3(8.9-13.9)$ & $10.0(7.4-17.0)$ & 0.852 & $8.2(6.4-11.1)$ & $78(63-116)$ & 0878 \\
\hline VVBC & $1.48(1.03-1.97)$ & $1.00(0.72-1.68)$ & 0.006 & $1.42(0.93-1.91)$ & $1.34(0.86-1.84)$ & 0.548 \\
\hline NLCR & $6.0(3.9-9.3)$ & $9.1(4.2-23.9)^{\#, \S}$ & 0.01 & $3.9(2.4-8.0)$ & $4.6(2.8-7.7)$ & 0.992 \\
\hline $\begin{array}{l}\text { Postoperative } \\
\text { COVID (+) patients, } \\
\text { n (\%) }\end{array}$ & $4(0.2)$ & - & & $2(0.4)$ & - & \\
\hline $\begin{array}{l}\text { ICU requirement, } \mathrm{n} \\
(\%)\end{array}$ & $28(2.3)$ & $18(46.2)$ & $<0.001$ & $9(2.0)$ & $5(20.0)$ & $<0.001$ \\
\hline Mortality, n (\%) & $0(0.0)$ & $1(2.6)$ & 0.805 & $0(0.0)$ & $1(3.6)$ & 0.898 \\
\hline
\end{tabular}

CAD, coronary arterial disease; COPD, chronic obstructive pulmonary disease; CRF, chronic renal failure; CVA, cerebrovascular accident; DM, diabetes mellitus; HCovP, hospitalized COVID patients; ICU, intensive care unit, NLCR, neutrophillymphocyte count ratio; IP, intervention patients; SP, surgical patients;WBC, white blood cell

\#, wilcoxon signed rank exact tests; $\S, p=0.005$ (preoperative NLCR \& postoperative NLCR) 


\begin{tabular}{|c|c|c|c|c|c|c|c|}
\hline & $\begin{array}{l}S P \\
(n=43)\end{array}$ & $\begin{array}{l}\text { IP } \\
(n=30)\end{array}$ & $\begin{array}{l}\text { Total }(\mathrm{SP}+ \\
\text { IP) } \\
(\mathrm{n}=73)\end{array}$ & $\begin{array}{l}\text { HCovP } \\
(n=1884)\end{array}$ & $\mathrm{p}_{1}$ & $\mathrm{p}_{2}$ & $\mathrm{p}_{3}$ \\
\hline Age, years & $\begin{array}{l}55(43- \\
66)\end{array}$ & $\begin{array}{l}50(10- \\
65)\end{array}$ & $56(45-66)$ & $\begin{array}{l}52(38- \\
67)\end{array}$ & 0.832 & 0.103 & 0.231 \\
\hline Male, $\mathrm{n}(\%)$ & $28(66.7)$ & $16(53.3)$ & $44(61.1)$ & $\begin{array}{l}1035 \\
(54.9)\end{array}$ & 0.132 & 1.000 & $0.303)$ \\
\hline ASA Classification>2, n (\%) & $7(16.3)$ & $13(43.3)$ & $20(27.4)$ & $35(1.9)$ & $<0.001$ & $<0.001$ & $<0.001$ \\
\hline \multicolumn{8}{|l|}{ Comorbidities, n (\%) } \\
\hline Hypertension & $12(27.9)$ & $17(56.7)$ & $29(39.7)$ & $206(10.9)$ & $<0.001$ & $<0.001$ & $<0.001$ \\
\hline DM & $4(9.3)$ & $6(20.0)$ & $10(13.7)$ & $114(6.1)$ & 0.353 & 0.006 & 0.007 \\
\hline CRF & $0(0.0)$ & $3(10.0)$ & $3(4.1)$ & $32(1.7)$ & 0.394 & 0.007 & 0.121 \\
\hline CAD & $4(9.3)$ & $10(33.3)$ & $14(19.2)$ & $77(4.1)$ & 0.083 & $<0.001$ & $<0.001$ \\
\hline Immunosuppressive drug & $20(46.5)$ & $24(80.0)$ & $44(60.3)$ & $3(0.2)$ & $<0.001$ & $<0.001$ & $<0.001$ \\
\hline COPD & $4(9.3)$ & $8(26.7)$ & $12(16.4)$ & $37(2.0)$ & $<0.001$ & $<0.001$ & $<0.001$ \\
\hline Smoking & $16(37.2)$ & $5(16.7)$ & $21(28.8)$ & $318(16.9)$ & $<0.001$ & 1.000 & 0.007 \\
\hline CVA & $0(0.0)$ & $2(6.7)$ & $2(2.8)$ & $18(0.1)$ & 0.525 & 0.032 & 0.131 \\
\hline $\begin{array}{l}\text { Preoperative COVID (+) patients, } \\
\mathrm{n}(\%)\end{array}$ & $39(90.7)$ & $28(93.3)$ & $64(88.9)$ & \multirow[t]{8}{*}{-} & \multirow[t]{8}{*}{-} & \multirow[t]{8}{*}{-} & \multirow[t]{8}{*}{-} \\
\hline CU requirement n $(\%)$ & $18(41.9)$ & $5(17.9)$ & $23(35.9)$ & & & & \\
\hline MV reguirement & $10(55.6)$ & $3(60.0)$ & $13(56.5)$ & & & & \\
\hline MV duration, days & $\begin{array}{l}16(12- \\
24)\end{array}$ & $13(2-28)$ & $14(10-27)$ & & & & \\
\hline & & $15(2-26)$ & $17(8-26)$ & & & & \\
\hline LUS-ILU, Uays & 22) & \multirow{3}{*}{$\begin{array}{l}19(17- \\
30) \\
1(3.6)\end{array}$} & $22(20-31)$ & & & & \\
\hline \multirow{2}{*}{ Mortality } & \multirow{2}{*}{$\begin{array}{l}26(22-32) \\
1(2.6)\end{array}$} & & $2(3.1)$ & & & & \\
\hline & & & & & & & \\
\hline \multirow{2}{*}{$\begin{array}{l}\text { Postoperative COVID (+) } \\
\text { patients, } \mathrm{n}(\%)\end{array}$} & $4(9.3)$ & $2(6.7)$ & $6(8.2)$ & \multirow[t]{4}{*}{-} & \multirow[t]{4}{*}{-} & \multirow[t]{4}{*}{-} & \multirow[t]{4}{*}{-} \\
\hline & $1(25.0)$ & $1(50.0)$ & $2(33.3)$ & & & & \\
\hline 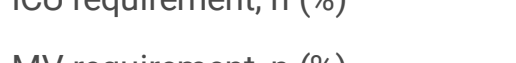 & $1(25.0)$ & $1(50.0)$ & $2(33.3)$ & & & & \\
\hline Mortality & $0(0.0)$ & $(0.0)$ & $(0.0)$ & & & & \\
\hline ICU requirement, n (\%) & $19(44.2)$ & $6(20.0)$ & $23(31.5)$ & $460(24.4)$ & 0.033 & 0.443 & 0.044 \\
\hline Mortality, n (\%) & $1(2.3)$ & $1(3.3)$ & $2(2.7)$ & $57(3.0)$ & 0.809 & 1.000 & 0.904 \\
\hline
\end{tabular}

CAD, coronary arterial disease; COPD, chronic obstructive pulmonary disease; CRF, chronic renal failure; CVA, cerebrovascular accident; DM, diabetes mellitus; HCovP, hospitalized COVID patients; ICU, intensive care unit, IP, intervention patients; SP, surgical patients

$\mathrm{p}_{1}$, SP\&HCovP ; $\mathrm{p}_{2}$, IP\&HCovP ; $\mathrm{p}_{3}$, Total\&HCovP 
Table 5. Infected patients and healthcare workers ratios

\begin{tabular}{|llll|}
\hline & $\begin{array}{l}\text { SP } \\
(\mathrm{n}=1273)\end{array}$ & $\begin{array}{l}\text { IP } \\
(\mathrm{n}=476)\end{array}$ & $\begin{array}{l}\text { HCovP } \\
(\mathrm{n}=1884)\end{array}$ \\
\hline Infected patients, $\mathrm{n}(\%)$ & $4(0.3)$ & $2(0.4)$ & - \\
\hline Infected healthcare workers, $\mathrm{n}(\%)$ & $17(1.0)$ & $72(3.8)$ \\
\hline Anesthesiologist, $\mathrm{n}(\%)$ & $3(0.2)$ & & - \\
\hline Anesthesia technician, $\mathrm{n}(\%)$ & $2(0.1)$ & & - \\
\hline Surgeon, $\mathrm{n}(\%)$ & $6(0.5)$ & - & $42(2.2)$ \\
\hline Doctor, $\mathrm{n}(\%)$ & - & $4(0.8)$ & $30(1.6)$ \\
\hline Nurse, $\mathrm{n}(\%)$ & $1(0.1)$ & $1(0.2)$ & \\
\hline CoVID-19 prevalence for hospital healthcare workers, $(\%)$ & 2.4 & & \\
\hline
\end{tabular}

HCovP, hospitalized COVID patients; IP, intervention patients; SP, surgical patients 